\title{
Assistência à saúde dos cuidadores de idosos com doença de Alzheimer: uma revisão integrativa
}

\author{
Health care of caregivers of elderly \\ with alzheimer's disease: an \\ integrative review
}

Fisisenectus . Unochapecó Ano 3, n. 1 - Jan/Jun. 2015 p. $19-28$

Rosane Paula Nierotka. rosanenier@unochapeco.edu.br

Fisioterapeuta. Mestre em Envelhecimento Humano pela Universidade de Passo Fundo - UPF. Docente do curso de graduação em fisioterapia da Universidade Comunitária da Região de Chapecó - Unochapecó.

Marilene Rodrigues Portella. portella@upf.br

Enfermeira. Doutora em Enfermagem pela Universidade Federal de Santa Catarina (UFSC). Docente do Programa de Mestrado em Envelhecimento Humano/PPGEH na Universidade de Passo Fundo. Líder do Grupo de pesquisas Vivencer CNPq/UPF.

Adriana Remião Luzardo. adriana.luzardo@uffs.edu.br Enfermeira. Doutora em Enfermagem pela Universidade Federal de Santa Catarina (UFSC). Docente na Universidade Federal da Fronteira Sul (UFFS) - Campus Chapecó.

\section{Resumo}

O objetivo do presente estudo é conhecer as evidências acerca da assistência à saúde dos cuidadores de idosos com Doença de Alzheimer. Trata-se de um estudo de abordagem qualitativa que adota uma Revisão Integrativa da Literatura (RI). A pesquisa bibliográfica eletrônica foi realizada nas bases de dados: Literatura Latino-Americana e do Caribe em Ciências da Saúde (LILACS), Scientific Electronic Library Online (SciELO), Base de Dados de Enfermagem (BDENF) e Pubmed com os seguintes descritores: Cuidadores, Doença de Alzheimer, Assistência e Cuidado. Combinação dos termos boolianos (and, or), os critérios de inclusão foram pesquisas qualitativas e quantitativas, revisão de literatura, relatos de experiência, programas e políticas de saúde. Já os critérios de exclusão foram: as publicações de trabalhos duplicados, cartas, editoriais, resumos em anais de eventos ou periódicos, comentários, ensaios, teses, dissertações, trabalho de conclusão de curso, livros, materiais publicados em outros idiomas que não Inglês, Espanhol e Português. Cabe mencionar que foi delimitado o período de 2002 a 2013 para coleta de dados e que a busca pela literatura ocorreu entre dezembro de 2012 e janeiro de 2013. Após a etapa da RI, vinte e um trabalhos foram selecionados e lidos na íntegra. Os resultados foram agrupados em quatro categorias: Sobrecarga e falta de informações dos cuidadores; Benefícios dos programas de assistência aos idosos e seus cuidadores; Remodelação e necessidade de políticas públicas e programas de assistência aos cuidadores. Observamos que ainda existem muitas lacunas na produção cientifica sobre esse assunto, ou seja, ainda faltam pesquisas e estudos que possam repercutir em ações eficazes a essa população

\section{Palavras-chave}

Cuidadores. Doença de Alzheimer. Assistência. Cuidado. 


\begin{abstract}
The objective is to know the evidence about the health care of caregivers of elderly with Alzheimer's disease. A qualitative study adopting an Integrative Literature Review (IR). The electronic literature search was performed in the databases: Literature Latin American and Caribbean Health Sciences (LILACS), Scientific Electronic Library Online (SciELO), Database of Nursing (BDENF), and PubMed using the following keywords: caregivers of Alzheimer's Disease Care and assistance. And as a combination of boolean terms (and, or). Inclusion criteria were qualitative and quantitative research, literature review, experience reports, programs and health policies and exclusion criteria, the publications of duplicate papers, letters, editorials, abstracts in conference proceedings or journals; comments; trials; theses; dissertations; completion of course work; books; materials published in other languages were not English, Spanish and Portuguese. The period 2002 to 2013 for data collection was delimited. The search for literature occurred between December 2012 and January 2013 After going through the steps of RI, blackjack works were selected and read fully, the results were grouped into four categories. Overload and lack of information from caregivers; Benefits of programs to assist the elderly and their caregivers; Remodeling and need for public policies and programs of assistance to caregivers. We note that there are still many gaps in the scientific literature on this subject, so that effective action can pass on this population.
\end{abstract}

\title{
Keywords
}

Caregivers. Alzheimer's disease. Assistance. Care.

\section{Introdução}

$\infty \times \infty \times \infty \times \infty \times \infty \times \infty \times \infty \times \infty \times \infty \times \infty \times \infty \times \infty)$

A Ataxia de Friedreich, descrita pela primeira veNa atualidade é crescente a presença dos longevos em nosso meio, seja pelas mudanças ocorridas no perfil demográfico, com a queda da fecundidade e mortalidade ou pelo avanço científico e desenvolvimento das tecnologias na assistência à saúde, os quais permitiram o controle das doenças infectocontagiosas ${ }^{1}$. Países como o Brasil têm testemunhado mudanças na pirâmide etária. Com crescente número de pessoas acima dos 60 anos ${ }^{2,3}$, dados do último censo revelam um contingente de aproximadamente 20.590.599 de idosos, representando $10,8 \%$ da população total $^{3,4}$, o que caracteriza a inversão da pirâmide etária.

Durante o processo de envelhecer é comum o aparecimento de doenças crônicas neurodegenerativas e demenciais, que podem ser caracterizadas pela presença de déficits progressivos que afetam principalmente a função cognitiva, com destaque para a perda de memória que interfere nas atividades desempenhadas pelo indivíduo ${ }^{5}$.

Nos países desenvolvidos, a prevalência de demência dobra a cada cinco anos e normalmente varia de $3 \%$ para indivíduos com 70 anos de idade e $20-30 \%$ àqueles de 85 anos $^{6}$. Sena e Gonçalves ${ }^{7}$ destacam que a Doença de Alzheimer (DA) é a forma mais comum de demência entre as pessoas acima de 65 anos, representando um percentual de $50 \%$ a $60 \%$ e estima-se que no mundo cerca de 15 milhões de pessoas sofrem da doença.

A DA compromete a integridade física, mental e social, acarretando manifestações cognitivas e neuropsiquiátricas que resultam na deficiência progressiva e incapacidade do indivíduo ${ }^{8}$, gerando dependência e necessidades de cuidados $^{9}$. Dentre as alterações decorrentes da patologia podemos ressaltar a perda de memória em curto prazo; dificuldade na fluência verbal, funções cognitivas, habilidades viso-motoras e destreza para manusear objetos e ferramentas ${ }^{10}$, havendo progressão dessas alterações conforme o agravamento da doença.

A necessidade de cuidados no transcurso da doença, conforme sua progressão, torna-se cada vez mais complexa, fazendo com que o idoso necessite de ajuda para a realização de quase todas as suas atividades do dia a dia, gerando múltiplas demandas de cuidado. Nesse contexto, diante da diversidade de cuidados rotineiros, surge a necessidade de uma assistência diária

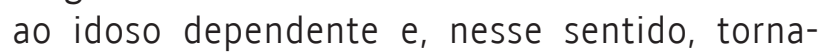


se imperativo que uma pessoa desempenhe a função de cuidar.

No Brasil, os cuidados ao idoso são realizados preponderantemente no ambiente familiar, muitas vezes sendo executado por um de seus membros. Surge, assim, o papel do cuidador como uma figura que, em geral, é uma mulher, membro da família, que assume, além das tarefas domésticas, o cuidado da pessoa, sem estar preparado para essa função ${ }^{11}$. Mesmo que várias responsabilidades sejam compartilhadas, quando uma pessoa assume o cuidado do idoso na situação de dependência no ambiente doméstico, muitas vezes, o resultado é a sobrecarga e sofrimento físico e psíquico ${ }^{12}$.

As dificuldades vivenciadas no processo de cuidar são frequentes e pouco visíveis ao contexto social, pois os cuidadores, na maioria das vezes, prestam um cuidado ininterrupto, desassistido e solitário, potencialmente gerador de estresse e sobrecarga ${ }^{13}$. Os desgastes gerados pela atividade cuidativa poderiam ser amenizados por uma assistência efetiva e qualificada caso fosse ofertado aos cuidadores alternativas capazes de minimizar o impacto dessa doença tanto na vida destes quanto na de sua família?.

Essa condição de sobrecarga do cuidador e de mudanças nos papéis sociais dos envolvidos no ato de cuidar, somado ao grau de dependência do idoso para quase todas as suas Atividade de Vida Diária (AVDs) e Atividades Instrumentais de Vida Diária (AIVDs) gera desconforto ao cuidador, que, sem apoio, fica exposto às situações de adoecimento.

Os serviços de saúde nem sempre estão preparados para responder à essa demanda e, além disso, se mostram pouco eficientes em termos de recursos públicos para atender as pessoas que vivem em condições vulneráveis. Desenvolver estratégias rotineiras que auxiliem as famílias a planejarem os momentos de cuidado, desde o diagnóstico até o agravamento da doença, não somente na durabilidade de grupos e programas de apoio é fundamental para minimizar os impactos negativos que 0 ato de cuidar pode gerar na saúde do cuidador.

Um estudo realizado por Guedea et $\mathrm{al}^{14}$ observou que o apoio social oferecido para ajudar nas necessidades dos cuidadores, sejam físicas, emocionais ou de informação, garantiu ao cuidador um suporte na realização das tarefas de cuidado, o que influenciou seu bem-estar e amenizou as tensões geradas pela sobrecarga cuidativa.

Os programas e grupos de apoio que existem nem sempre oferecem um atendimento de qualidade e experiências vivenciadas mostram essa falta de eficiência. Um exemplo está na falta de acesso dos cuidadores aos grupos de apoio, pois dificuldades, como o deslocamento ou até mesmo por não terem onde deixar o idoso dependente, fazem com que eles não consigam usufruir do atendimento oferecido, o que nos leva a refletir sobre como está sendo aferida a assistência a essa população.

Diante dessas reflexões, acredita-se que ao realizar uma Revisão Integrativa de Literatura (RI) seja possivel identificar os estudos que vêm tratando da problemática da assistência à saúde dos cuidadores de idosos com DA, contribuindo, assim, para análise das produções recentes acerca da temática do cuidador e suscitando recomendações para espaços de investigação ainda não explorados. Dessa forma, ao analisar os estudos que vêm sendo realizados, poder-se-á instigar a produção de mais pesquisas sobre esse tema tão relevante, mostrando, cada vez mais, a importância de políticas públicas efetivas que deem conta dessas necessidades.

A partir da problemática apresentada o presente estudo propõe-se a responder a seguinte pergunta de pesquisa: Como tem ocorrido a assistência à saúde dos cuidadores de idosos com Doença de Alzheimer a partir das evidências de estudos publicados entre 2002 a 2013? Nesse sentido objetivou-se com este estudo: Conhecer as evidências acerca da assistência à saúde dos cuidadores de idosos com Doença de Alzheimer de estudos publicados de 2002 a 2013.

\section{Metodologia}

$\infty \times \infty \times \infty \times \infty \times \infty \times \infty \times \infty \times \infty \times \infty \times \infty \times \infty \times \infty \times \infty$

Trata-se de um estudo com abordagem qualitativa que buscou conhecer as produções sobre assistência à saúde dos cuidadores de 
idosos com Doença de Alzheimer. Adotou-se, como técnica de pesquisa, a Revisão Integrativa da Literatura (RI) proposta por Ganong ${ }^{15}$.

Segundo Silveira ${ }^{16}$, em virtude da complexidade de informações na área da saúde, tornou-se imprescindível o desenvolvimento de mecanismos, cientificamente embasados que propiciem aos profissionais a melhor utilização das evidências apresentadas em inúmeros estudos. Nesse cenário, a revisão integrativa emerge como um método que proporciona a síntese do conhecimento e a incorporação da aplicabilidade de resultados de estudos significativos na prática.

Esse método constitui-se em um instrumento da Prática Baseada em Evidências (PBE). A $\mathrm{PBE}$, cuja origem atrelou-se ao trabalho do epidemiologista Archie Cochrane, caracteriza-se por uma abordagem voltada ao cuidado clínico e ao ensino fundamentado no conhecimento e na qualidade da evidência. Envolve, pois, a definição do problema clínico, a identificação das informações necessárias, a condução da busca de estudos na literatura e sua avaliação crítica, a identificação da aplicabilidade dos dados oriundos das publicações e a determinação de sua utilização para o paciente ${ }^{17}$.

Para Ganong ${ }^{15}$, todas as etapas dessa revisão estão alicerçadas em uma estrutura formal de trabalho em que se inclui o estabelecimento de critérios bem-definidos sobre a coleta de dados, análise e apresentação dos resultados desde o início do estudo, a partir de um protocolo de pesquisa previamente elaborado e validado.

0 presente estudo adotou as seis etapas indicadas por Ganong ${ }^{15}$ para a constituição da revisão integrativa da literatura: seleção da pergunta de pesquisa; definição dos critérios de inclusão de estudos e seleção da amostra; representação dos estudos selecionados em formato de tabelas, considerando todas as características em comum; análise crítica dos achados, identificando diferenças e conflitos; interpretação dos resultados; e relato, de forma clara, da evidência encontrada. Tais etapas constituíram o protocolo para realização deste estudo que está detalhado a seguir.

A busca pela literatura ocorreu entre dezembro de 2012 e janeiro de 2013. As bases de dados
Literatura Latino-Americana e do Caribe em Ciências da Saúde (LILACS), a Scientific Electronic Library Online (SciELO), a Base de Dados de Enfermagem (BDENF) e a PubMed contemplaram um universo de 223 estudos. Como estratégias de busca foram utilizados os seguintes descritores e suas combinações nas línguas portuguesa e inglesa (Descritores em Ciências da Saúde - DeCS/ MeSH: Cuidadores, Doença de Alzheimer).

Os critérios de inclusão foram definidos a partir de artigos de pesquisas qualitativas, quantitativas, estudos mistos sobre o tema, revisões de literatura, relatos de experiência, programas, políticas de saúde e estudos que apresentaram os descritores selecionados. Como critérios de exclusão temse: as publicações de trabalhos duplicados, cartas, editoriais, resumos em anais de eventos ou periódicos, comentários, ensaios, teses, dissertações, trabalho de conclusão de curso, livros e materiais publicados em outros idiomas que não fossem inglês, espanhol e português. Foi delimitado um período temporal de 2002 a 2013 para a avaliação dos materiais coletados.

Após selecionar os 223 estudos iniciou-se a leitura flutuante destes com análise dos títulos e resumos, considerando os critérios de pertinência e consistência do conteúdo. Nesse sentido, foram selecionados 49 estudos completos para análise mais aprofundada. Em concordância com o terceiro item das etapas estabelecidas na RI para a extração dos dados dos estudos selecionados, foi construída uma tabela contendo colunas que descriminavam as seguintes informações: título, ano de publicação, objetivo, descritores, sujeitos da pesquisa, abordagem/tipo de estudo, principais resultados, conclusão do pesquisador, referências utilizadas e revista publicada. Os dados foram agregados conforme a identificação de semelhanças e diferenças, compondo um achado que foi interpretado e sustentado com outras literaturas.

A análise dos dados foi realizada de forma descritiva, permitindo ao leitor conhecer a literatura sobre o tema investigado e ter a possibilidade de identificar lacunas para 0 desenvolvimento de novas pesquisas, bem como eventuais intervenções necessárias. Segundo 
Minayo ${ }^{18}$, os dados foram analisados preconizando os seguintes passos:

1 - Ordenação dos dados: etapa de transcrição das observações, releitura do material e organização dos relatos, compondo um corpo teórico.

2 - Classificação dos dados: processo de leitura exaustiva e repetida dos textos, constituição de um "corpus" de comunicação, leitura transversal de cada corpo como recorte de "unidade de registro" e enxugamento dos dados mais relevantes.

3 - Análise final e elaboração das categorias analíticas: fase em que se consideram os objetivos da pesquisa e os temas que emergem das observações e faz-se a articulação dos dados com o referencial teórico, definindo as categorias analíticas.

\section{Resultados e Discussão}

$\infty \times \infty \times \infty \times \infty \times \infty \times \infty \times \infty \times \infty \times \infty \times \infty \times \infty \times \infty)$

Foi encontrado um total de 223 citações, por meio da busca dos descritores e da leitura de títulos e resumos. Assim, 139 publicações foram identificadas na base de dados PubMed, 58 na base LILACS, 17 artigos na BDENF e nove estudos na SCIELO.

Após refinamento, respeitando critérios de inclusão/exclusão, foram selecionados 49 estudos, os quais foram lidos na íntegra e em profundidade. Desses artigos 21 foram relevantes para a proposta estudada. Também foram incluídos artigos que descreviam a assistência aos cuidadores de idosos que apresentavam outro tipo de demência que não fosse o Alzheimer, considerando a relevância das informações e as demandas semelhantes dos cuidados executados pelos cuidadores.

Em relação aos sujeitos do estudo, a maioria eram cuidadores informais, destacando-se os familiares, porém, alguns estudos mencionaram, também, cuidadores formais e outros não deixavam delineado o tipo de cuidador. Houve certa diversificação em relação aos periódicos em que os estudos foram publicados. Destacaram-se 11 publicações nacionais e dez internacionais.
Dos estudos selecionados, a maioria contemplava abordagem quantitativa (11), representando $52,38 \%, 5$ estudos $(23,80 \%)$ foram realizados com abordagem qualitativa, 2 estudos (9,52 \%) eram híbridos (quanti e qualitativo), 2 estudos (9,52\%) tinha enfoque na revisão bibliográfica e $1(4,76 \%)$ era relato de experiência.

Das 21 produções científicas, os resultados foram agrupados em três categorias por temas de convergência, conforme apresentado em anexo no Quadro 1. Na categoria "sobrecarga e falta de informações dos cuidadores" foram identificados 11 estudos, nos quais evidenciou-se o despreparo dos cuidadores em relação ao ato de cuidar. Estes, geralmente, assumem o ato de cuidar sem informações necessárias quanto à progressão da doença e ao modo adequado de realizar os cuidados, o que gera grandes sobrecargas físicas, psicológicas, financeiras e sociais.

0 ato de cuidar geralmente traz aos cuidadores um variado repertório de sentimentos (angústia, culpa, inversão de papéis, ira, agressividade, sofrimento, solidão, tristeza e medo). Além de conviver com a dependência do portador em quase ou todas suas AVDs e AIVDs e passar por situações consideradas constrangedoras como dar banho e trocar de roupa, o cuidador, muitas vezes, também enfrenta a falta de apoio e reconhecimento por parte do idoso cuidado e da sua família ${ }^{19,20}$.

Cuidar de idosos com algum tipo de demência pode comprometer vários anos de vida de uma pessoa, devido à lenta progressão da doença. Esses cuidados podem gerar emoções, doenças, dificuldades, desgaste, tensões, estresse e, além de afetar todo o sistema cardiovascular, também pode afetar a função imunológica e alteração do sono, mas, sobretudo, exige muita dedicação ${ }^{21,23}$.

Os dados apresentados no estudo de Silva; Passos e Barreto ${ }^{24}$ evidenciaram que o grau de sobrecarga dos cuidadores foi de leve a moderado $(51,7 \%)$. A questão emocional e física esteve bastante presente, sendo que dentre os problemas de saúde autorreferidos, os mais prevalentes foram dores nas costas $(63,8 \%)$, problemas articulares $(60,3 \%)$, colesterol alto $(51,7 \%)$ e hipertensão arterial (44,8\%). Os medicamentos mais utilizados foram os anti-hipertensivos (38,9\%) e antidepressivos (31,5\%). 
As sobrecargas que os cuidadores enfrentam são as mais variadas possíveis, a questão financeira é outro fator que afeta a família, pois os gastos com a doença aumentam com a progressão desta ${ }^{13}$. Enfrentam, também, a sobrecarga de tarefas e de compromissos, pois o acompanhamento desses pacientes é contínuo e ininterrupto e, com a ausência de apoio institucional, da própria família e da sociedade em geral, faz com que o cuidador deixa de lado seu autocuidado em prol do cuidado do próximo ${ }^{25}$.

Sabe-se que cuidar de um idoso com DA é algo desafiador. Os cuidadores, na maioria das vezes, ao se depararem com seu familiar, que antes apresentava domínio para quase todas as suas atividades e que no momento necessita de ajuda para desempenhar atividades mínimas de seu cotidiano, acabam deixando de lado sua saúde. No entanto, os programas de assistência são benéficos nesse ponto, ajudando tanto o idoso, quanto o cuidador em vários aspectos demonstrados na categoria a seguir.

A categoria "benefícios dos programas de assistência aos idosos e seus cuidadores", emergiu de 13 publicações. Os estudos trouxeram um grande destaque sobre a importância de programas como os grupos de ajuda mútua e de apoio que são oferecidos aos idosos com DA e aos seus cuidadores.

Segundo Santana; Almeida e Savoldi ${ }^{26}$ os grupos de assistência são momentos de acolhimento e atenção, onde a troca de experiências e apoio mútuo entre familiares, cuidadores e a comunidade em geral, com assuntos e atividades voltados a uma determinada patologia, como a DA, constituem-se em espaços em que os cuidadores e idosos recebem apoio e informações sobre a demência e sua progressão, bem como treinamento e orientação familiar para auxiliá-los a lidar com as tensões e dificuldades da função que exercem.

A participação em grupos de assistência, em que eram oferecidos programas como: reabilitação cognitiva, assistência do computador, formação cognitiva, terapia da fala, terapia ocupacional, arte-terapia, treinamento físico, fisioterapia e estimulação cognitiva com leituras e jogos de lógica, trouxe benefícios tanto aos idosos quanto aos cuidadores ${ }^{27}$. Ao integrarem-se a esses grupos, os idosos com DA apresentaram certa estabilização nos déficits cognitivos e funcionais e nos sintomas neuropsiquiátricos, melhorando sua autonomia funcional 28 .

Para os cuidadores, os grupos de assistência têm fundamental importância, pois, após essa integração, os cuidadores apresentaram uma diminuição da sobrecarga física, mental e social, as atividades de cuidado tornaram-se mais agradáveis, houve melhora na capacidade de comunicação e sua relação com o idoso, bem como no desempenho das atividades diárias desse cuidador. As informações divulgadas pelo grupo atenderam as necessidades dos cuidadores que passaram a desenvolver o cuidado com mais segurança e calma, evitando negligências, além de perceber a importância de zelar por sua saúde física e emocional, garantindo, assim, um cuidado de qualidade ${ }^{20,29}$.

Segundo Dugglebyet et al., ${ }^{30}$ os cuidadores devem buscar a assistência juntamente com os profissionais da saúde, no apoio da família e amigos e na facilidade de acesso a serviços e informações apropriadas. O sentimento gerado em quem recebe a assistência é de muita gratidão e reconhecimento, pois após receber uma assistência qualificada é perceptível a melhora significativa na qualidade de vida dos cuidadores, redução do estresse e aumento do seu bem-estar em geral, além de sensibilizar para o autocuidado.

Outro fator interessante observado nessa revisão, segundo Diel et al. ${ }^{31}$, foi em relação aos benefícios dos grupos de assistência para a comunidade em geral. Pessoas sem relatos de experiência com a DA, para ajudar esses cuidadores e idosos que enfrentam a demência, ao perceberem o quanto a DA é um problema sério e de difícil cuidado, demonstraram interesse pela prevenção e conscientização quanto à necessidade de conhecimentos para identificar precocemente a doença.

Apesar dos benefícios que apresentam esses grupos de assistência, vários desses estudos demonstraram que alguns programas têm curta duração e atendem só uma parcela da população necessitada, deixando muitas famílias que têm 
dificuldades no acesso às unidades de atendimento sem assistência.

Por diversos motivos, alguns núcleos familiares não conseguem acessar os cuidados oferecidos pelos programas citados e, nesse sentido, emergiu a terceira categoria: "remodelação e necessidade de políticas públicas e programas de assistência aos cuidadores", identificada em 13 publicações.

A necessidade de assistência aos idosos com DA e seus cuidadores é vista como algo prioritário, oferecendo informações, orientações sobre a patologia e sobre o ato de cuidado, diminuindo, assim, a grande sobrecarga apresentada no decorrer dessa função. Mas, para dar conta dessas necessidades há muito a ser feito. Alguns estudos nos mostram alternativas, como a criação de políticas públicas com programas efetivos e duradouros que deem conta e que consigam inserir toda essa população de cuidadores, proporcionando uma melhor qualidade de vida e de cuidado 9 , 22, 32

Inserir essa população vai além de oferecer programas de assistência, mas também pensar em como estão sendo divulgados esses programas. Para que se tenha sucesso em uma estratégia, é necessário que esta atinja e traga benefícios a todos os necessitados ou a boa parte destes, porem, segundo Roque et al. ${ }^{29}$, deve-se pensar em como vamos levar essa assistência para que todos consigam e tenham interesse em buscá-la.

Atualmente existem alguns programas direcionados aos cuidadores de idosos com DA, como a Associação Brasileira de Alzheimer (ABRAz). A ABRAz oferece a mais de 20 anos a assistência a familiares e cuidadores de pessoas com DA e outras demências, contribuindo para a superação de dificuldades enfrentadas desde o momento do diagnóstico até as etapas mais avançadas da doença. Esse grupo de apoio é um espaço de encontro, aprendizagem e troca de experiências oferecidas, no qual os participantes têm a oportunidade de refletir sobre a tarefa de cuidado sob novas perspectivas, a encontrar novas estratégias para superar dificuldades e descobrir novas formas de lidar com o cotidiano modificado ${ }^{33}$.

Além da ABRAz outras formas de assistência existem, como o fornecimento de medicamentos para o Alzheimer, centros de referências e grupos de apoio. Essa realidade, no entanto, ainda é permeada por muitas limitações. Conforme mencionado, para garantir o acesso à assistência, além da criação de novas políticas públicas, é necessário reestruturar a forma de divulgação destas e pensar como torná-las acessíveis a todas as famílias, tendo em vista as dificuldades enfrentadas pelo ato de cuidar de um idoso com DA ${ }^{29,34}$.

A necessidade de apoio a essa população, atualmente, é um assunto abrangente e visto com urgência para a atenção à saúde ${ }^{26}$. A remodelação para atender essa população deve ocorrer desde a formação profissional, para que, ao se deparar com um idoso com diagnóstico sugestivo de Alzheimer, os profissionais já tenham um olhar voltado para o seu cuidador, proporcionando uma melhor qualidade de vida e cuidado com essa família.

\section{Considerações Finais}

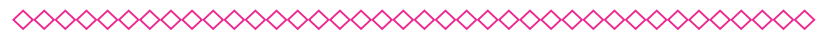

Neste estudo, a análise da produção científica sobre a assistência aos cuidadores de idosos com DA, permitiu ampliar os conhecimentos sobre os estudos que vêm sendo produzidos em relação a essa temática tão relevante do ponto de vista das demandas de saúde pública.

Verificou-se que os estudos analisados reportaram-se para o tema sobre as demandas e as sobrecargas dos cuidadores de idosos, tanto físicas, quanto emocionais, financeiras ou sociais. A preocupação dessas análises é a pouca repercussão sobre a questão de como resolver ou apoiar esses cuidadores e de apontar ou relatar estratégias exitosas que minimizam o impacto dessa situação.

Constatou-se nos estudos que ao participar dos programas de assistência os idosos e seus cuidadores tornam-se beneficiados de alguma forma. Por outro lado, os referidos programas ainda não são capazes de oferecer assistência contínua e de qualidade a todos os necessitados.

Reitera-se que o ato de cuidar de idosos com DA envolve situações de complexidade frente às necessidades e dificuldades enfrentadas. 
Portanto, como apresentado em quase todos os estudos analisados, há evidências acerca dos déficits consideráveis em relação à assistência aos cuidadores, bem como uma necessidade crescente da criação de programas de assistência abrangentes e integradores acerca das demandas dos idosos e seus respectivos cuidadores.

Observou-se, por meio desta revisão, que ainda existem muitas lacunas na produção cientifica sobre esse assunto, espaços ainda inexplorados que podem repercutir em ações públicas mais efetivas. Novos estudos são necessários, não só para apontar a urgência de uma maior assistência aos cuidadores, mas também para apontar caminhos governamentais que oportunizem apoio e atenção às famílias.

\section{Referências}

$\infty \times \infty \times \infty \times \infty \times \infty \times \infty \times \infty \times \infty \times \infty \times \infty \times \infty \times \infty \times \infty \times \infty)$

1. Almeida L, Azevedo RCS, Reiners AAO, Sudré MRS. Cuidado realizado pelo cuidador familiar ao idoso dependente, em domicílio, no contexto da estratégia de Saúde da Família. Texto contexto enferm. 2012;21(3):543-548.

2. Bechara FT, Santos SMS. Efetividade de um programa fisioterapêutico para treino de equilíbrio em idosos. Saúde e Pesquisa. 2008;1(1):15-20.

3. Instituto Brasileiro de Geografia e Estatística (IBGE). Sinopse do Senso Demográfico de 2010. Rio de Janeiro; 2011.

4. Küchemann, BA. Envelhecimento populacional, cuidado e cidadania: velhos dilemas e novos desafios. Sociedade e Estado. 2012;27(1):165180.

5. Gallucci NJ, Tamellini MG, Forlenza OV. Diagnóstico diferencial das demências. Rev Psiq Clín. 2005;32(3):119-130.

6. Fagundes SD, Silva MT, Thees MFRS, Pereira MG. Prevalence of dementia among elderly Brazilians: a systematic review. Sao Paulo Med. J. 2011 Jan;129(1):46-50.

7. Sena ELS, Gonçalves LHT. Vivências de familiares cuidadores de pessoas idosas com doença de Alzheimer - Perspectiva da filosofia de Merleau-Ponty. Texto contexto - enferm. 2008 June;17(2):232-240.

8. Luzardo AR, Gorini MIPC, Silva Ana Paula SS. Características de idosos com doença de Alzheimer e seus cuidadores: uma série de casos em um serviço de neurogeriatria. Texto contexto enferm. 2006;15(4):587-594.

9. Camacho ACLF, Brum AKR, Sá SPC, Lindolpho MC, Valente GSC, Louredo DS. Programa para cuidadores de idosos com demência: um relato de experiência. Ver. Pesqui. Cui. Fundam. 2012;4(2):2898-2904.

10. Sereniki A, Vital MABF. A doença de Alzheimer: aspectos fisiopatológicos e farmacológicos. Rev. psiquiatr. Rio Gd. Sul. 2008;30(1 Suppl).

11. Scortegagna HM, Portella MR, Pinheiro GML. A doença crônica no curso da vida. In: Pomatti DM, Bettinelli LA. Doenças crônicas: múltiplas abordagens. Passo Fundo: Berthier, 2009, p. 219234.

12. Agich G. Dependência e autonomia na velhice: um modelo ético para o cuidado de longo prazo.

São Paulo: Loyola, Centro Universitário São Camilo, 2008.

13. Luzardo AR, Waldman BF. Atenção ao familiar cuidador do idoso com doença de Alzheimer. Rev. Acta Scientiarum. 2004 Jan-Jun;26(1):b135-45.

14. Guedea MTD, Damacena FA, Carbajal MMM, Marcobich PO, Hernández GA, Lizárraga LV, Flores EI. Necessidades de apoio social em cuidadores de familiares idosos mexicanos. Psicologia \& Sociedade. 2009;21(2):242-249.

15. Ganong LH. Integrative reviews of nursing research. Res Nurs Health 1987 Mar; 10(1):1-11.

16. Silveira RCCP. O cuidado de enfermagem e o cateter de Hickman: a busca de evidências. 2005. Dissertação (Mestrado em Enfermagem Fundamental) - Escola de Enfermagem de Ribeirão Preto, Universidade de São Paulo, Ribeirão Preto, 2005. 
17. Galvão CM, Sawada NO, Trevizan MA. Revisão sistemática: recurso que proporciona a incorporação das evidências na prática da enfermagem. Rev. Latinoam. Enfermagem. 2004 Mai-Jun;12(3):549-56.

18. Minayo MCS. O desafio do conhecimento: pesquisa qualitativa em saúde. 8 ed. São Paulo: Hucitec, 2004. 269 p. (Saúde em debate 46) ISBN 85-271-0181-5.

19. Fonseca AM, Soares E. O cuidador e os cuidados ao portador de doença de Alzheimer: contribuições à enfermagem. Rev. Mineira Enferm. 2008;12(4):501-507.

20. Agüero MA, Estrada AF, Ruiz JRL, Blanco MA, Álvarez RG. Capacitación a personas cuidadoras de pacientes con demencia. MEDISAN. 2009 Abr;13(2).

21. Varela G, Varona L, Anderson K, Sansoni J: Alzheimer's care at home: a focus on caregivers strain. Prof Inferm 2011;64:113-117.

22. DeFries EL, McGuire LC, Andresen EM, Brumback BA, Anderson LA. Caregivers of older adults with cognitive impairment. Preventing chronic disease, public health research, practice and policy. Prev Chronic Dis. 2009;6(2).

23. Freitas ICC, Paula KCC, Soares JL, Parente ACM. Convivendo com o portador de Alzheimer: perspectivas do familiar cuidador. Rev. bras. enferm. 2008;61(4):508-513.

24. Silva CF, Passos VMA, Barreto SM. Frequência e repercussão da sobrecarga de cuidadoras familiares de idosos com demência. Rev. Bras. Geriatr. Gerontol. 2012;15(4):707-731.

25. Lima Junior FAV, Silva WHS, Costa FA. 0 impacto de acidente vascular cerebral no cotidiano de cuidadores familiares. Estud. interdiscip. envelhec. 2012;17(2):251-264.

26. Santana RF, Almeida KS, Savoldi NAM. Indicativos de aplicabilidade das orientações de enfermagem no cotidiano de cuidadores de portadores de Alzheimer. Rev. esc. enferm. USP. 2009;43(2):459-464.
27. Viola LF, Nunes PV, Yassuda MS, Aprahamian I, Santos FS, Santos GD et al. Effects of a multidisciplinar cognitive rehabilitation program for patients with mild Alzheimer's disease. Clinics. 2011;66(8):1395-1400.

28. Carbone G, Barreca F, Mancini G, Pauletti $\mathrm{G}$, Salvi V, Vanacore $\mathrm{N}$, et al. A home assistance model for dementia: outcome in patients with mild-to-moderate Alzheimer's disease after three months. Ann Ist Super Sanita. 2013;49:34-41.

29. Roque FP, Ortiz KZ, Araújo MSC, Bertolucci PHF. Eficácia de treinamento de estratégias comunicativas a cuidadores de pacientes com demência. Pró-Fono R. Atual. Cient. 2009 Set;21(3):225-230.

30. Duggleby WD, Swindle J, Peacock S, Ghosh S. A mixed methods study of hope, transitions, and quality of life in family caregivers of persons with Alzheimer's disease. BMC Geriatr. 2011;11:88.

31. Diel L, Forster LMK, Kochhann R, Chaves MLF. Sociodemographic profile and level of burden of dementia patients' caregivers who participate in a support group. Dement Neuropsychol. 2010;4(3):232-237.

32. Perdomo MP. Orientaciones para una mejor atención de los ancianos con Alzheimer en la comunidad. Rev. Haban. Cienc. méd. 2008;7(4).

33. Associação Brasileira de Alzheimer (ABRAz). Disponivel em: http://www.abraz.com.br/. Acessado em: 23/10/2013 as 10:00 horas.

34. Domingues MARC, Santos CF, Quintans JR. Doença de Alzheimer: o perfil dos cuidadores que utilizam o serviço de apoio telefônico da ABRAz Associação Brasileira de Alzheimer. Mundo Saúde. 2009;33(1):161-169. 


\section{Anexos}

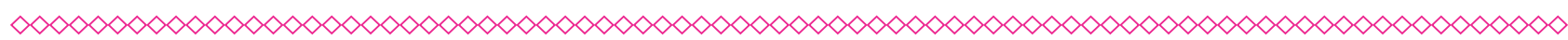

Quadro 1 - Categorias analíticas encontradas na revisão da literatura - 2013

\begin{tabular}{|c|}
\hline Categorias Analíticas \\
\hline 01: Sobrecarga e falta de informações dos cuidadores \\
\hline Categoria 02: Benefícios dos programas de assistência aos idosos e seus cuidadores \\
\hline Categoria 03: Remodelação e necessidade de políticas públicas e programas de \\
assistência aos cuidadores
\end{tabular}

Fonte: Elaborado pelos Autores 